\title{
An analytical model for YORP and Yarkovsky effects with a physical thermal lag
}

\begin{abstract}
E. Mysen
Institute of Theoretical Astrophysics, University of Oslo, PO Box 1029 Blindern, 0315 Oslo, Norway

e-mail: eirik.mysen@astro.uio.no

Received 16 November 2007 / Accepted 21 March 2008

ABSTRACT

Asteroids absorb solar radiation, which is later re-emitted. In this paper, an analytic approach for the description of the effects of this thermal emission on the rotation and orbit of an asteroid of unspecified shape is presented. The theory is connected directly to the physics of the problem, and the important results caused by a delayed thermal response of the surface are, therefore, parameterized by the fundamental surface properties of the asteroid. Overall results of previous numerical studies are recovered and an application to the elongated and irregularly-shaped asteroid Eros shows correspondence. The dependency of the derived differential equations on the dynamical variables is explicit and simple. We argue that if the transport of asteroids within the main belt is caused by thermal emission, then there is a preference for the shapes of Earth orbit crossing, regolith-covered asteroids.
\end{abstract}

Key words. celestial mechanics - minor planets, asteroids - methods: analytical

\section{Introduction}

Asteroids in orbit around the Sun reflect and absorb solar radiation. Absorbed photons are thermalized and then emitted at longer wavelengths. The process determines the local temperature at the surface of an asteroid, and is dependent on the local insolation pattern as well as the conduction of heat in the body. If conduction is significant, the local temperature, and therefore also the thermal emission of radiation, peaks in the afternoon, i.e., after the point when the Sun was highest on the sky as seen from the particular place on the asteroid surface.

The emission of photons leads to a recoil force which even for a spherical body (Vokrouhlický 1998) changes the size of its orbit, also called the Yarkovsky effect. Thermal emission of absorbed solar radiation by asteroids has been recognized as an important transport mechanism of asteroids to the resonances of the main belt (Farinella et al. 1998; Bottke et al. 2007), whereupon they may end up as Earth orbit crossing objects. Since the Yarkovsky force leading to the changes in heliocentric orbit is dependent on a delayed thermal response of the body's surface, its effects are coupled to the rotation state of the asteroid.

To have a proper description of the Yarkovsky effects, we also need to understand the rotation of asteroids. The torques from the recoil force due to thermal emission have been recognized to be important (Rubincam 2000), especially when it comes to changes in the rotation period and angle between the asteroid orbit plane and asteroid equator, called the equatorial obliquity. From the numerical studies of Rubincam (2000) and Vokrouhlický \& Căpek (2002), it is clear that the ability of thermal emission to change the rotation rate and obliquity of asteroids, which we here define as YORP effects, is, in the limit of no heat conduction, sensitively dependent on the irregularity of the asteroid's shape. That is, there are no YORP effects for asteroids shaped like triaxial ellipsoids with immediate thermal response to solar radiation. Later on, the numerical studies of Čapek \& Vokrouhlický (2004) demonstrated that heat conduction changes the thermal emission's ability to alter the asteroid obliquity, but not the rotation rate.

Recently, analytical studies of these phenomena have been initiated. For instance, analytic theory can strengthen previous results, uncover new effects, and reveal more precisely which parameters of the system determine important results. The development of analytic theories for YORP effects have posed exceptional challenges since the asteroid must, in a unified theory, be irregular and have a delayed thermal response.

Significant steps in this direction were made by Breiter et al. (2007), which showed that a thermally-delayed emission of radiation on biaxial ellipsoids can change the bodies' obliquities, but not their rotation rates. Some additional key results were uncovered in Nesvorný \& Vokrouhlický (2007) for near-spherical, but irregular shapes. An elaborate attempt is also given by Scheeres (2007), but it does not present differential equations with a clear structure for the obliquity, e.g., as should be required by an analytic theory. Instead, the structure of the differential equations have to be resolved for each particular asteroid shape in these, therefore, (semi-)numerical investigations. As a result, the Scheeres (2007) approach has the benefit of numerical studies, namely that fewer approximations need be made, and some aspects of the problem are, therefore, not lost.

The challenge of this problem is mainly to describe analytically the insolation pattern and the terminator on an asteroid surface of irregular shape. In Mysen (2008), we circumvented the problem with a simple approximation of the insolation pattern, encompassing the need for a detailed characterization of the transition from day to night on the asteroid. Overall results from previous studies were recovered. However, as in all the referenced analytic studies, we implemented the thermal lag by delaying the response of the force with respect to noon at the asteroid, possibly yielding unphysical results. We connect the Mysen (2008) approach directly to the physics of the problem, which allows for a proper evaluation of the different components' relative importance. 


\section{Heat transport of an asteroid}

A common approach for the calculation of temperature on an asteroid's surface is the one-dimensional heat equation for material that does not change properties with temperature and location

$\varrho C \frac{\partial T}{\partial t}=K \frac{\partial^{2} T}{\partial \zeta^{2}}$

where $T$ is the temperature. Above, $\varrho$ is the mass density, $C$ the specific heat capacity and $K$ the heat conductivity, while $\zeta$ is the depth along the line normal to the asteroid surface element. As indicated by Harris \& Lagerros (2002), it is acceptable to use a one-dimensional approximation if the penetration depths of the heat waves are much smaller than the size of the asteroids. That is, the use of Eq. (1) sets lower limits for the diameter of the body to which the equation is applied, limits which will be evaluated later on.

Equation (1) should be combined with the boundary condition at the surface, $\zeta=0$, of the asteroid

$\Psi(T)-K \frac{\partial T}{\partial \zeta}=S_{\odot} \hat{\epsilon}, \quad \hat{\epsilon}=\Theta(\cos z) \cos z$,

where the term $\Psi=\sigma T^{4}$ represents the energy sink due to thermal emission. Here, $\Theta$ is the step function, which is one for positive and zero for negative arguments, and the insolation $\hat{\epsilon}$ is a function of the local zenith angle $z$ of the Sun at the surface element in question. The solar flux is given by

$S_{\odot}=\frac{L_{\odot}}{4 \pi R_{\odot}^{2}}$

where $L_{\odot}$ is the solar luminosity and $R_{\odot}$ is the asteroid's heliocentric distance. The emissivity and visual albedo have for convenience been set to one and zero, respectively.

As discussed in Mysen (2007) and Mysen (2008), the insolation function $\hat{\epsilon}$ can be approximated by the expression

$\hat{\epsilon} \approx \epsilon \equiv \vartheta_{0}+\vartheta_{1} \cos z+\vartheta_{2} \cos ^{2} z$.

With $\vartheta_{0}=0.106, \vartheta_{1}=0.500$, and $\vartheta_{2}=0.4244$, the average deviation between $\hat{\epsilon}$ and $\epsilon$ is only 0.034 (Mysen 2007; Mysen $2008)$, which should be compared to the maximum $\hat{\epsilon}=1$. Note that the effect of the step function $\Theta$ has already been included at this level, and that when we later need to integrate over the surface of the asteroid to find the total recoil force or torques, the integration can be done over the whole surface, and not only the day side. That is, the dynamical variables have been removed from the integration boundaries, an approach which here is adopted from Neishtadt et al. (2002) and Neishtadt et al. (2003). If the results should be sensitively dependent on terms that are not included in the fairly good approximation, Eq. (4), the physicality of the results can be questioned as these small variations in $\hat{\epsilon}$ perhaps can be considered arbitrary for a real asteroid.

In Eq. (4), an expression for $\cos z$ is needed. To this end, let the body-fixed unit vectors along the principal axes, the axes around which a body can execute uniaxial rotation (Goldstein 1980), be given by $\boldsymbol{u}_{x_{i}}$. An outwards pointing unit vector $\boldsymbol{n}$ normal to a surface element of the asteroid can then be written in terms of its direction cosines $\gamma_{x_{i}}$,

$\mathrm{d} \boldsymbol{S}=\mathrm{d} S \boldsymbol{n}=\mathrm{d} S \sum_{i=1}^{3} \gamma_{x_{i}} \boldsymbol{u}_{x_{i}}$

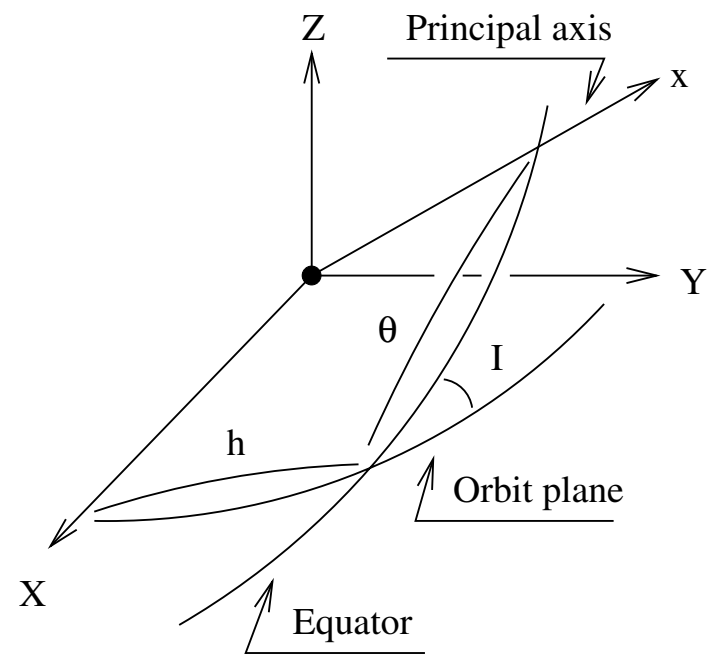

Fig. 1. Spin orientation parameters for an asteroid.

with $\mathrm{d} S$ as the surface area of the element. If the mass centered unit vector $\boldsymbol{u}_{\odot}$ of the Sun is written in the same way

$\boldsymbol{u}_{\odot}=\sum_{i=1}^{3} \gamma_{\odot, x_{i}} \boldsymbol{u}_{x_{i}}$

one gets

$\cos z=\boldsymbol{n} \cdot \boldsymbol{u}_{\odot}=\sum_{i=1}^{3} \gamma_{x_{i}} \gamma_{\odot, x_{i}}$.

The $\gamma_{\odot, x_{i}}$ functions again are dependent on the spin orientation and orbit parameters of the asteroid. If we, for the moment, define the orbit plane of the asteroid as our reference plane, the direction cosines of the Sun can be written (Mysen 2008)

$\gamma_{\odot, x_{i}}=\gamma_{\odot, x_{i}}(u, h, \theta, I)$

where $h, \theta$, and $I$ are the spin orientation parameters of Fig. 1. Note that rotation is defined to be uniaxial around the principal $z$-axis. Above, $u=\bar{\omega}+f$ where $f$ is the Sun's true anomaly and $\bar{\omega}$ its argument of pericenter, both measured counterclockwise in the $X Y$-plane from the $X$-axis. Now, due to planetary perturbations, the orbit plane of the asteroid undergoes significant variations. The reference $X$-axis above can then be defined by the cross section between an inertial reference plane and the orbit plane. Later, we present a differential equation for an angle $I^{*}$ in the form $\dot{I}^{*}=\dot{I}^{*}\left(I, \theta^{*}\right)$ where the angles $I^{*}$ and $\theta^{*}$ correspond to $I$ and $\theta$, but are measured relative to the inertial plane. We then make the reasonable approximation $I \approx I^{*}$ such that $\dot{I}^{*}=\dot{I}^{*}\left(I^{*}, \theta^{*}\right)$. Exceptions can arise when there are spin-orbit resonances (Vokrouhlický et al. 2003). The difference between $I$ and $I^{*}$ will be notationally suppressed.

From Eqs. (4), (7), and (8), the insolation can with some basic trigonometric manipulation be reduced to the form

$\epsilon=\sum_{j=0}^{2} \sum_{k=-2}^{2} \epsilon_{j k}(h, I, \bar{\omega}, \boldsymbol{n}) \cos \left(j \theta+k f-\varphi_{j k}\right)$,

where $\boldsymbol{n}$ is the unit vector normal to the surface element in question, see Eq. (5). Note that the equation above is valid for any shape as long as self-shadowing is not taken into account. Due to 
energy dissipation caused by inelastic distortions in the asteroid (Burns \& Safronov 1973; Efroimsky 2001), asteroids larger than a certain size (Mysen 2006) are expected to rotate around their axes of maximum moment of inertia, or so-called short-axis. In the following, it will also be assumed that the heliocentric orbit is circular so that both $f$ and $\theta$ change linearly in time,

$f=n\left(t-t_{0}\right), \quad \theta=\omega\left(t-t_{0}\right)$,

where $n$ is the rate of the orbit mean anomaly and $\omega$ the rate of the rotational phase $\theta$. If we substitute these expressions into Eq. (9) for the calculation of the thermal emission's dynamical effects, consistent with the iterative approach of first-order perturbation theory, one obtains

$\epsilon=\sum_{i} \epsilon_{i} \cos \left(\theta_{i}-\varphi_{i}\right), \quad \theta_{i}=\omega_{i}\left(t-t_{0}\right)$

yielding variability with different constant frequencies

$\omega_{i}=j \omega+k n$.

To obtain a general solution, the temperature is linearized around a constant $T_{0}$

$\Psi=\Psi_{0}+\Psi_{0}^{\prime} \Delta T, \quad \Psi_{0}=\Psi\left(T_{0}\right), \quad \Psi_{0}^{\prime}=\frac{\partial \Psi}{\partial T_{0}}$,

where by definition

$\frac{\partial T_{0}}{\partial \zeta}=\frac{\partial T_{0}}{\partial t}=0$

Substitution of the formal Eq. (11) into Eqs. (1) and (2) yields that if $\omega_{0} \equiv 0$, then

$\Psi_{0} \equiv \sigma T_{0}^{4}=S_{\odot} \epsilon_{0}, \quad \omega_{0} \equiv 0$.

For the variable temperature $\Delta T$ of Eq. (13), which is a function of both time $t$ and depth $\zeta$, one gets

$$
\begin{aligned}
\Delta T= & S_{\odot} \sum_{i \neq 0} \frac{\epsilon_{i}}{\sqrt{\beta_{i}^{2}+\Gamma_{i}^{2}}} \\
& \times \exp \left(-\tilde{\Gamma}_{i} \zeta\right) \cos \left(-\tilde{\Gamma}_{i} \zeta+\theta_{i}-\eta_{i}-\varphi_{i}\right)
\end{aligned}
$$

where

$\beta_{i}=\Psi_{0}^{\prime}+\Gamma_{i}, \quad \Gamma_{i}=K \tilde{\Gamma}_{i}=\sqrt{\omega_{i} / 2} \sqrt{\varrho C K}$.

The thermal lag angles $\eta_{i}$ are given by

$$
\cos \eta_{i}=\frac{\beta_{i}}{\sqrt{\beta_{i}^{2}+\Gamma_{i}^{2}}}, \quad \sin \eta_{i}=\frac{\Gamma_{i}}{\sqrt{\beta_{i}^{2}+\Gamma_{i}^{2}}}
$$

which go towards $\eta_{i} \rightarrow 45^{\circ}$ as $K \rightarrow \infty$. A measure of how deep a thermal wave penetrates is from Eq. (16)

$\zeta_{i}=\frac{1}{\sqrt{2} \tilde{\Gamma}_{i}}=\sqrt{\frac{K}{\varrho C \omega_{i}}}$,

known as the skin depth (Harris \& Lagerros 2002).

\section{Parameterization of YORP evolution}

The variation of solar gravity over the asteroid produces torques. If not close to the Sun where the rotational evolution may be chaotic (Skoglöv 1996), such gravity-gradients are not effective when it comes to changes in rotation rate and obliquity. The effects of thermal emission on these parameters are, therefore, of great interest. One should note that the forces connected to the direct absorption of photons do not lead to changes in the spin orientation or rotation rate of an irregular body with uniform surface properties (Rubincam 2000; Mysen 2006).

According to Lambert's law (Čapek \& Vokrouhlický 2004), the force acting on a surface element with area $\mathrm{d} S$ and unit normal vector $\boldsymbol{n}$ is from Eqs. (13) and (15)

$\mathrm{d} \boldsymbol{F}=-\frac{2}{3} \frac{\mathrm{d} S}{c} \Psi(T)=-\frac{2}{3} \frac{\mathrm{d} S}{c}\left(S_{\odot} \epsilon_{0}+\Psi_{0}^{\prime} \Delta T\right)$

where $\Delta T$ is evaluated at depth $\zeta=0$. The torque acting on the element with position $\boldsymbol{r}$ relative to the mass center of the asteroid is then

$\mathrm{d} \boldsymbol{\tau}=\boldsymbol{r} \times \mathrm{d} \boldsymbol{F}, \quad \boldsymbol{r}=\sum_{i=1}^{3} x_{i} \boldsymbol{u}_{x_{i}}$.

For later applications, it is also useful to define the torque component

$\hat{\boldsymbol{\tau}} \equiv \boldsymbol{n} \times \boldsymbol{r}, \quad \hat{\tau}_{x_{i}}=\sum_{j, k=1}^{3} \varepsilon_{i j k} \gamma_{x_{j}} x_{k}$

where $\varepsilon_{i j k}$ is the antisymmetric Levi-Civita symbol of the cross product.

\subsection{Rotation rate}

From Mysen (2008), the change in the magnitude $G$ of the asteroid's rotational angular momentum, or spin, is given by the differential equation

$\dot{G}=\tau_{z}$

where $\tau_{z}$ is the torque component along the principal $z$-axis, the axis of rotation. To remove the short-period oscillations of this variable, we average Eq. (23) over unperturbed motion, consistent with first-order, non-resonant perturbation theory (Fernandes \& Sessin 1989)

$\langle A\rangle=\lim _{T \rightarrow \infty} \frac{1}{T} \int_{0}^{T} \mathrm{~d} t A=\frac{1}{(2 \pi)^{2}} \int_{0}^{2 \pi} \mathrm{d} \theta \int_{0}^{2 \pi} \mathrm{d} u A$.

For the last transition above, the orbit has been assumed to be circular. Already now, since Eq. (23) only contains the phases $\theta_{i}$ of Eq. (11) through the torque $\tau_{z}\left(\theta_{i}\right)$, it is possible with the use of Eq. (24) to conclude that thermal lag does not influence the secular evolution of the rotation rate, consistent with previous results mentioned in the introduction. To be more specific, the contribution from a surface element on an asteroid with area $\mathrm{d} S$ is

$\mathrm{d}\left\langle\frac{\dot{G}}{G}\right\rangle=\left\langle\frac{\mathrm{d} \tau_{z}}{G}\right\rangle=2 \chi \mathrm{d} S \hat{\tau}_{z} \epsilon_{0}, \quad \chi \equiv \frac{1}{3 G} \frac{S_{\odot}}{c}$.

Equations (4), (7), and (8) yield

$$
\begin{aligned}
\epsilon_{0}=\langle\epsilon\rangle= & \vartheta_{0}+\frac{\vartheta_{2}}{2}\left(1-\frac{1}{2} \sin ^{2} I\right) \\
& +\gamma_{z}^{2} \frac{\vartheta_{2}}{2}\left(\frac{3}{2} \sin ^{2} I-1\right),
\end{aligned}
$$


remembering that $\gamma_{z}$ is the component along the principal $z$-axis of the asteroid of the surface element's unit normal vector $\boldsymbol{n}$. Integrating over the whole surface results in

$$
\begin{aligned}
\left\langle\frac{\dot{G}}{G}\right\rangle= & 2 \chi \Lambda_{0}\left[\vartheta_{0}+\frac{\vartheta_{2}}{2}\left(1-\frac{1}{2} \sin ^{2} I\right)\right] \\
& +\chi \Lambda_{2} \vartheta_{2}\left(\frac{3}{2} \sin ^{2} I-1\right)
\end{aligned}
$$

with asteroid shape parameters

$\Lambda_{i} \equiv \oint \mathrm{d} S \gamma_{z}^{i} \hat{\tau}_{z}$

From basic calculus, $\Lambda_{0}=0$ also for irregular bodies (see Mysen 2007), while it can be demonstrated that the same result applies to $\Lambda_{2}$ if the surface is that of an ellipsoid. That is, the ability of thermal emission to change the rotation rate of asteroids is sensitively dependent on the shape of the asteroid. These are the same equations and results as in Mysen (2008), but rederived here within the framework of the more physical approach.

\subsection{Equatorial obliquity}

The differential equation for the calculation of the obliquity is also given in Mysen (2008)

$(\cos I)=\frac{\sin I}{G}\left(\tau_{y} \cos \theta+\tau_{x} \sin \theta\right)$.

For a torque, which is typically dependent on the angle $\theta \equiv \theta_{1}$ through the thermally delayed $w_{1}=\theta_{1}-\eta_{1}$ with $\eta_{1}$ from Eq. (18), it is more convenient to operate with $w_{1}$ explicitly in Eq. (29)

$$
\begin{aligned}
(\cos I) \sim & \tau_{y}\left(w_{1}\right) \cos \theta+\tau_{x}\left(w_{1}\right) \sin \theta \\
= & \cos \eta_{1}\left[\tau_{y}\left(w_{1}\right) \cos w_{1}+\tau_{x}\left(w_{1}\right) \sin w_{1}\right] \\
& -\sin \eta_{1}\left[\tau_{y}\left(w_{1}\right) \sin w_{1}-\tau_{x}\left(w_{1}\right) \cos w_{1}\right] .
\end{aligned}
$$

Inserting the torques from the force Eq. (20) into Eq. (30) and taking note of the fact that from Eq. (16)

$$
\left\langle\Delta T\left(w_{1}\right) \cos w_{1}\right\rangle=\langle\Delta T(\theta) \cos \theta\rangle=S_{\odot} \frac{\langle\epsilon \cos \theta\rangle}{\sqrt{\beta_{1}^{2}+\Gamma_{1}^{2}}},
$$

yields

$$
\begin{aligned}
\mathrm{d}\langle(\cos I)\rangle= & 2 \chi \frac{\Psi_{0}^{\prime} \sin I}{\sqrt{\beta_{1}^{2}+\Gamma_{1}^{2}}} \mathrm{~d} S\left[\cos \eta_{1}\left(\hat{\tau}_{y} \epsilon^{c}+\hat{\tau}_{x} \epsilon^{s}\right)\right. \\
& \left.-\sin \eta_{1}\left(\hat{\tau}_{y} \epsilon^{s}-\hat{\tau}_{x} \epsilon^{c}\right)\right] .
\end{aligned}
$$

Equations (4), (7), and (8) again yield

$\epsilon^{c} \equiv\langle\epsilon \cos \theta\rangle=-\frac{\vartheta_{2}}{2} \gamma_{y} \gamma_{z} \sin I \cos I$

and

$\epsilon^{s} \equiv\langle\epsilon \sin \theta\rangle=-\frac{\vartheta_{2}}{2} \gamma_{x} \gamma_{z} \cos I \sin I$.

Inserting these into Eq. (32) results in

$$
\langle(\cos I)\rangle=\chi \vartheta_{2} \sin ^{2} I \cos I\left(\kappa_{1}^{s} \mathrm{~d} \chi_{s}-\kappa_{1}^{c} \mathrm{~d} \chi_{c}\right) .
$$

The parameters dependent solely on the asteroid's shape are

$\mathrm{d} \chi_{s}=\mathrm{d} S\left(\gamma_{x} \gamma_{z} \hat{\tau}_{y}-\gamma_{y} \gamma_{z} \hat{\tau}_{x}\right)$ and

$\mathrm{d} \chi_{c}=\mathrm{d} S\left(\gamma_{y} \gamma_{z} \hat{\tau}_{y}+\gamma_{x} \gamma_{z} \hat{\tau}_{x}\right)=-\mathrm{d} S \gamma_{z}^{2} \hat{\tau}_{z}$

where the geometrical $\hat{\tau}_{x_{i}}$ are those of Eq. (22).

The thermal $\kappa_{1}^{c, s}$ functions, on the other hand, are dependent on the obliquity $I$ and fundamental properties of the asteroid as well as the surface normal vector $n$

$\kappa_{1}^{c}=\frac{\Psi_{0}^{\prime} \cos \eta_{1}}{\sqrt{\beta_{1}^{2}+\Gamma_{1}^{2}}}=\frac{\Psi_{0}^{\prime} \beta_{1}}{\beta_{1}^{2}+\Gamma_{1}^{2}}$

and

$\kappa_{1}^{s}=\frac{\Psi_{0}^{\prime} \sin \eta_{1}}{\sqrt{\beta_{1}^{2}+\Gamma_{1}^{2}}}=\frac{\Psi_{0}^{\prime} \Gamma_{1}}{\beta_{1}^{2}+\Gamma_{1}^{2}}$.

One way to parameterize them is through the function

$\lambda_{1} \equiv \frac{\Psi_{0}^{\prime}}{\Gamma_{1}}=\frac{4 \sigma T_{0}^{3}}{\Gamma_{1}}=\frac{4 \sigma}{\Gamma_{1}}\left(\frac{S_{\odot}}{\sigma}\right)^{3 / 4} \epsilon_{0}^{3 / 4} \equiv \bar{\lambda}_{1} \epsilon_{0}^{3 / 4}$,

from Eq. (15) where $\epsilon_{0}$ of Eq. (26) can be used, so that

$\kappa_{1}^{c}=\frac{\lambda_{1}\left(\lambda_{1}+1\right)}{\left(\lambda_{1}+1\right)^{2}+1}$

and

$\kappa_{1}^{s}=\frac{\lambda_{1}}{\left(\lambda_{1}+1\right)^{2}+1}$

Since it is natural for an analytic theory to present a general form of differential equations, it is particularly important to resolve the thermal functions' dependencies on the obliquity $I$. One easy way to do this is to expand $\kappa_{1}^{c, s}$ in powers of $\gamma_{z}$, the $z$-component of the surface normal vector $\boldsymbol{n}$. Investigations show that developments to order $\gamma_{z}^{2}$ only yield quite precise approximations to the actual $\kappa_{1}^{c, s}$. We do not adopt more precise expansions since they are not essential to the points made. Instead, the convention

$\epsilon_{0}^{3 / 4} \longrightarrow 0.3$

in Eqs. (41) and (42) is used, see Eq. (40). The error of this assumption is mapped in Fig. 2 for the worst-case $\gamma_{z}=1$, an error which is seen to drop considerably as $\gamma_{z}$ decreases. Accordingly, it is possible that the conclusions that are made with assumption Eq. (43) can be somewhat modified near obliquities $I \sim 0$, $I \sim \pi / 2$ and $I \sim \pi$, if there are many surface elements of the asteroid with $\gamma_{z} \sim 1$. The value Eq. (43) is chosen so that the relative differences, like those plotted in Fig. 2, are as small as possible for a wide range of obliquities and $\bar{\lambda}$. Note that $\bar{\lambda}_{1}$ is not indexed in the plot since it is considered as the argument of $\kappa^{s}$. That is, the results are not dependent on the particular frequency used. The function $\kappa^{s}$ is not indexed for the same reason.

Before proceeding, the behavior of $\kappa_{1}^{c, s}$ should be emphasized. Included as Fig. 3 is a plot of these two factors as functions of $\bar{\lambda}_{1}$ of Eq. (40), using the approximation Eq. (43). As can be seen, while $\kappa_{1}^{c}$ steadily goes to zero with decreasing $\bar{\lambda}_{1}, \kappa_{1}^{s}$ has, with approximation Eq. (43), a peak in the range $2 \lesssim \bar{\lambda}_{1} \lesssim 15$ for which

$\kappa_{1}^{s}\left(2 \lesssim \bar{\lambda}_{1} \lesssim 15\right) \sim 0.2$.

In the same $\bar{\lambda}_{1}$ range, $0.2 \lesssim \kappa_{1}^{c} \lesssim 0.8$. 




Fig. 2. The plot shows the contours in $\bar{\lambda}$ and obliquity space on which $\left|\kappa^{s}-\kappa^{s}\left(\epsilon_{0}^{3 / 4}=0.3\right)\right| / \kappa^{s}$ is $0.25,0.28$, and 0.5 in a worst-case scenario of $\gamma_{z}=1$. The results for $\kappa^{c}$ are similar.

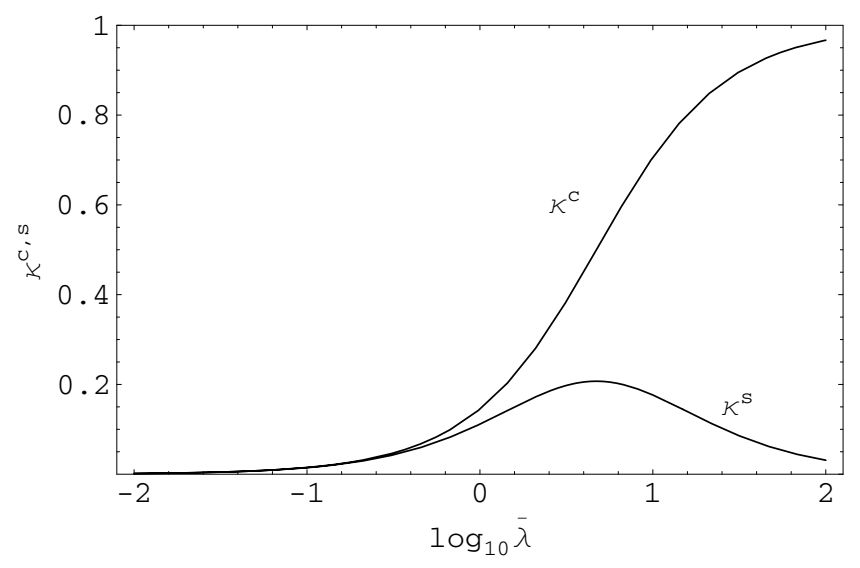

Fig. 3. The thermal $\kappa^{c, s}$ of Eqs. (41) and (42) are shown as functions of the parameter $\bar{\lambda}$ of Eq. (40), using the approximation Eq. (43).

\subsection{Analysis}

If we drop the brackets, which indicate that we are dealing with secular evolution or mean variables, from Eqs. (27) and (35) we obtain:

$\frac{\dot{G}}{G}=\chi \vartheta_{2} \Lambda_{2}\left(\frac{3}{2} \sin ^{2} I-1\right), \quad \Lambda_{2}=-\chi_{c}$

$(\cos I)=\chi \vartheta_{2}\left(\kappa_{1}^{s} \chi_{s}-\kappa_{1}^{c} \chi_{c}\right) \sin ^{2} I \cos I$

where the approximation Eq. (43) is implicitly assumed, and the $\kappa_{1}^{c, s}$ functions are, therefore, not dependent on $I$. These equations have the same general structure as those presented in Mysen (2008), but this time, the parameters have been connected to the fundamental properties of the asteroid. Note that there is no seasonal YORP effect, i.e., no dependency on the orbit period of the asteroid, which follows from the structures of Eqs. (23) and (29). Also, it must be stressed again that the equations above are valid for completely arbitrary shapes as long as the effects from selfshadowing are small.

To summarize the results of Mysen (2008), $\chi_{c}=-\Lambda_{2}$ is zero for a triaxial ellipsoid and its sign for real irregular asteroids is, therefore, dependent on the particular deviations of the irregular shape from that of a triaxial ellipsoid. If these deviations are random, the sign of $\chi_{c}$ is random, too. In the limit of no heat conduction, $\kappa_{1}^{c} \rightarrow 1$ and $\kappa_{1}^{s} \rightarrow 0$, it is then from Eq. (46) equally probable with obliquity equilibria at $I=\pi / 2$ in comparison to $I=0$ or $I=\pi$. In both types of equilibria, i.e., with spin axis or equator normal to the orbit, the asteroid will from Eq. (45) spin down in agreement with the overall results of Vokrouhlický \& Căpek (2002).

As heat conduction increases and from Eqs. (41) and (42), $\kappa_{1}^{c} \sim \kappa_{1}^{s} \sim \lambda_{1} / 2$, it is possible that the $\chi_{s}$ component can be of importance. If we adopt a sign for $\chi_{s}$ corresponding to that of a triaxial ellipsoid, which rotates around its shortest axis due to energy dissipation (Burns \& Safronov 1973; Efroimsky 2001), and from a number of numerical integrations is positive (Mysen 2008), then $I \rightarrow 0, \pi$ from Eq. (46). In these equilibria, the asteroids spin up or down dependent on the particular realization of $\Lambda_{2}$, Eq. (45), consistent with the overall results of Čapek \& Vokrouhlický (2004)

From Eq. (45), we see that the change in rotation rate goes to zero when $I \sim 55^{\circ}$ and $I \sim 125^{\circ}$, which has been pointed out to be the case for some shapes before (Breiter et al. 2007; Nesvorný \& Vokrouhlický 2007).

There are also a number of asteroid shapes reported in the literature with tendencies, which deviate from what is contained within the model Eqs. (45) and (46). Some tendencies can be due to the approximation Eq. (43), i.e., related to the fact that the same thermal lag is not global. It is also possible that the precisions of Eqs. (45) and (46) suffer from the linearization of the problem Eq. (13). Other types of stability than those of Eqs. (45) and (46) can be produced by self-shadowing, which has not been taken into account, or can originate from higher order terms of the expansion Eq. (4). That is, within the linear approximation, Eqs. (45) and (46) demonstrate that the deviations are caused by the fact that thermal delay is not constant across the asteroid, by self-shadowing or details of the interaction law.

Finally, a transition value for $\bar{\lambda}_{1}$ of Eq. (40) can be defined. That is, if $\chi_{c}$ happens to be positive and $\chi_{s}$ is positive, then the obliquity equilibria change nature at

$\bar{\lambda}_{t}=\frac{1}{0.3}\left(\frac{\chi_{s}}{\chi_{c}}-1\right)$

from Eqs. (40)-(42) and (46).

\section{The shape parameters}

Now, the properties of parameters Eqs. (28), (36), and (37) of Eqs. (45) and (46) will be explored. Partly due to the integrands' dependencies on higher orders of the surface normal components $\gamma_{x_{i}}$, the formulation of the integrals in terms of commonly known functions does not seem to be trivial for irregular surfaces. However, the reader should be aware of some basic properties, based on a number of specific regular shape realizations. First of all, the surface integral $\Lambda_{2}=-\chi_{c}$ is zero for triaxial ellipsoids because the integrand changes sign a number of times across the surface, variations which eventually sum up to zero. If the ellipsoid is biaxial, the integrand is zero everywhere on the surface. As long as ellipsoidal shapes rotating around their 


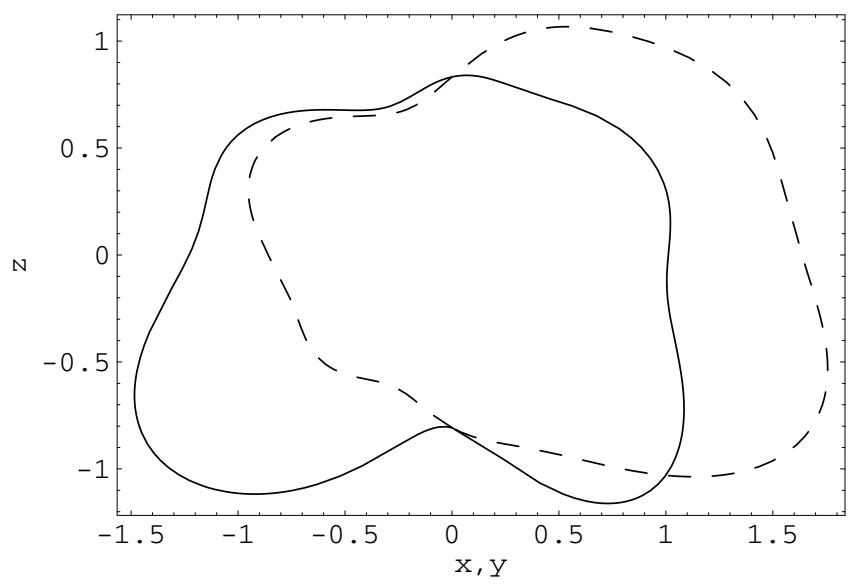

Fig. 4. Two cross sections of a realization of a Gaussian shape.

shortest axes are considered, $\chi_{s}$ is positive. For rotation around a triaxial ellipsoid's longest axis, $\chi_{s}$ is negative.

In other words, asteroids shaped like triaxial ellipsoids with uniform surface properties align their spin axes with the orbit normal if there is a delayed thermal response. The ellipsoidality of the asteroid's shape is responsible for this kind of alignment. This property can also to some extent be reasoned from Breiter et al. (2007), but we also demonstrate for an irregular shape, with a thermal lag that is parameterized in terms of the fundamental properties of the asteroid surface. That is, a random shape with a surface that does not correlate with the principal axes of the body has a $\chi_{s}$ with random sign and the rotation axis does not necessarily align itself with the orbit normal. Now, since the irregularity of the shape does not produce the sought generic result, it can be ruled out as a cause. We believe that this generic result, rotation axis alignment with the orbit normal, was obtained with the random shapes of Čapek \& Vokrouhlický (2004) since their principal axes were determined by their surfaces, as indicated in Vokrouhlický \& Căpek (2002). That is, relative to the axis of maximum moment of inertia, determined by the surface, the random shapes become oblate or ellipsoidal.

The question is then to what extent irregularities in the shape can alter the generic result, which from Eq. (46) is dependent on thermal delay. That is, as $\kappa_{1}^{c} \rightarrow 1$ and $\kappa_{1}^{s} \rightarrow 0$, the location of the obliquity equilibria are determined solely by the sign of $\chi_{c}$, which can be random.

\subsection{Gaussian shape}

To shed some light on this question, so-called Gaussian shapes (Muinonen 1998), where the logarithm of the radii are generated by normal statistics, are studied. First, a mean radius $r_{\mathrm{a}}$, radius standard deviation $\sigma_{\mathrm{a}}$, and the surface correlation angle are set to

$r_{\mathrm{a}}=1, \quad \sigma_{\mathrm{a}}=0.25, \quad \Gamma_{\mathrm{a}}=0.5$

based on the analysis of some Solar System bodies (Muinonen \& Lagerros 1998). Two cross sections of a realization are included as Fig. 4. The principal axes of the shape are predefined to coincide with axes of the coordinate system in which the shape is generated, and are, therefore, not corrected after the shape is realized. That is, it is not necessarily assumed that the mass density is constant, although constant mass density seemed to be a good assumption for the asteroid Eros (Miller et al. 2002).
Numerical integrations, based on a division of the shape's surface into 10000 elements as is done for all such integrations, yield

$\chi_{s}=0.304, \quad \chi_{c}=0.243, \quad \bar{\lambda}_{t}=0.84$

for the shape of Fig. 4. In this case, both $\chi_{c}$ and $\chi_{s}$ happened to be positive and the stability of the obliquity equilibria changes nature at the $\bar{\lambda}_{t}$ of Eq. (47) given above. This value should be compared to some possible ones for asteroids. At heliocentric distance $R_{\odot}=3 \mathrm{AU}$, a rotation period of $P=10 \mathrm{~h}$ and heat capacity $C=600 \mathrm{~J} \mathrm{~kg}^{-1} \mathrm{~K}^{-1}$, we obtain for regolith-covered (R), basaltic (B), and metal-rich (M) asteroids (Farinella et al. 1998)

$\bar{\lambda}_{1}(R)=7.9, \quad \bar{\lambda}_{1}(B)=0.12, \quad \bar{\lambda}_{1}(M)=0.021$

from Eq. (40), where the densities go from

$\varrho(R, B, M)=(1.5,3.5,8) \mathrm{g} \mathrm{cm}^{-3}$

and the heat conductivities as

$K(R, B, M)=(0.0015,2.65,40) \mathrm{W} \mathrm{m}^{-1} \mathrm{~K}^{-1}$.

That is, if the body is covered with regolith and $\bar{\lambda}_{1}>\bar{\lambda}_{t}$, the delay in the thermal response of the surface to solar radiation is not enough to align the asteroid's rotation axis with the orbit normal. Instead, the rotation axis seeks the orbit plane, an obliquity equilibrium in which the asteroid spins down, Eq. (45). If the body is metal-rich or basaltic, on the other hand, its rotation axis will align with the orbit normal since $\bar{\lambda}_{1}(B, M)<\bar{\lambda}_{t}$, although this occurs on a longer timescale due to the lower $\kappa_{1}^{c} \sim \kappa_{1}^{s} \sim \lambda_{1}(B, M) / 2$.

The skin depths Eq. (19) of the frequency given by the tenhour rotation period and material above are

$\zeta_{1}(R)=0.3 \mathrm{~cm}, \zeta_{1}(B)=9 \mathrm{~cm}$, and $\zeta_{1}(M)=22 \mathrm{~cm}$,

which should be much smaller than the size of the asteroid for the approach Eq. (1) to hold.

\subsection{Ellipsoidality}

Now, it will be investigated what happens when the shape gets slightly more ellipsoidal. For this purpose, the radius vector of the surface is set to a sum of two components, $\boldsymbol{r}=\boldsymbol{r}_{\mathrm{e}}+\boldsymbol{r}_{\mathrm{G}}$, where the first one is that of a triaxial ellipsoid with half-axes $a_{\mathrm{e}}=b_{\mathrm{e}}=0.4$ and $c_{\mathrm{e}}=0.1$. The second component $\boldsymbol{r}_{\mathrm{G}}$ represents a contribution from the Gaussian shape realized in the previous section, Fig. 4, but downscaled by a factor 0.7 . That is, one can expect the realized shape to be centered around a biaxial ellipsoid with half-axes $a_{\mathrm{e}}=b_{\mathrm{e}}=1.1$ and $c_{\mathrm{e}}=0.8$, roughly yielding the same volume as the pure Gaussian shape in the previous section. Figure 5 shows two cross sections of this shape. Numerical integrations yield

$\chi_{s}=0.615, \quad \chi_{c}=0.187, \quad \bar{\lambda}_{t}=7.6$

for the shape Fig. 5. As can be seen, $\chi_{s}$ is significantly increased so that $\bar{\lambda}_{1}(R)$ is almost lower than the transition value Eq. (47) .

Finally, the Gaussian shape of Fig. 4 is downscaled by a factor 0.3 and combined with an ellipsoid with half-axes $a_{\mathrm{e}}=1.2$, $b_{\mathrm{e}}=0.7$ and $c_{\mathrm{e}}=0.4$, the sum forming a shape centered around the ellipsoid $a_{\mathrm{e}}=1.5, b_{\mathrm{e}}=1.0$ and $c_{\mathrm{e}}=0.7$. That is, the final shape has roughly the same volume as those already studied, and is illustrated in Fig. 6. Accordingly,

$\chi_{s}=1.89, \quad \chi_{c}=-0.103, \quad$ and $\quad \bar{\lambda}_{t}=58$ 


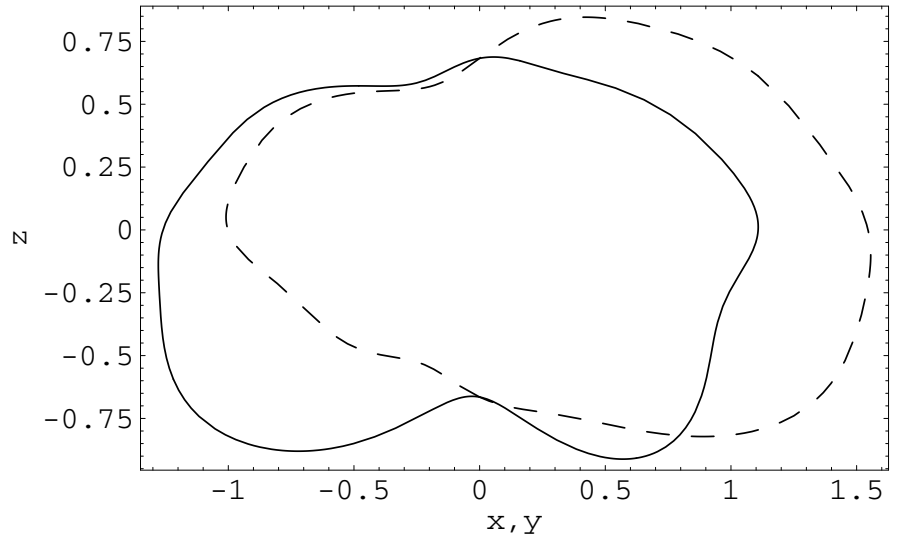

Fig. 5. This oblate shape is obtained by a radius vector, which is a superposition of a biaxial ellipsoid and a Gaussian realization.

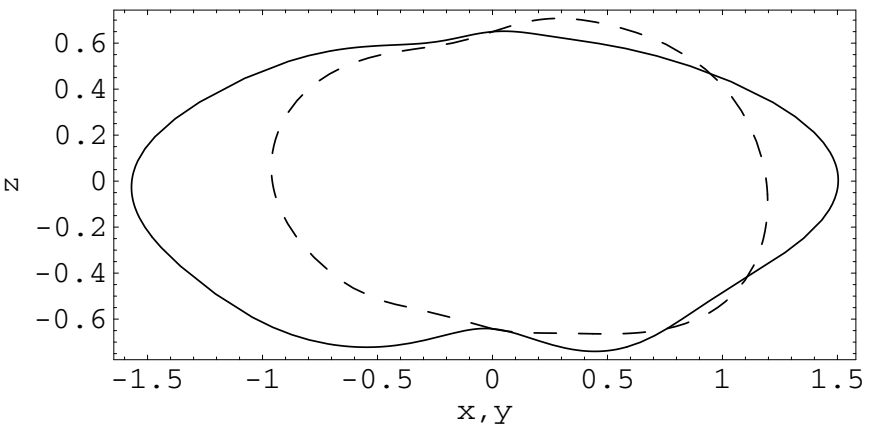

Fig. 6. This shape is obtained by a radius vector, which is a sum of a triaxial ellipsoid and a Gaussian realization.

where the very high transition value $\bar{\lambda}_{t}$ is calculated using the absolute value of the $\chi_{c}$ above. That is, it has been demonstrated that the absolute values for the shape parameters of a plausible random surface, based on analysis of a number of Solar System bodies, are smaller than the range of shape parameter values produced by plausible ellipsoidalities of the asteroid's body. However, it is possible that the Gaussian shape, which is used to demonstrate this point, is a statistically unlikely realization. A number of additional realizations studied in the same way as above indicates that this is not so.

If radius normalized values like Eq. (49), (54), and (55) are used in Eqs. (45) and (46), then these should be multiplied with a factor $r^{3}$ where $r$ is the asteroid radius. For instance, using the spin of a spherical body with bulk mass density $\varrho=2.5 \mathrm{~g} \mathrm{~cm}^{-3}$, the value

$\frac{\dot{G}}{G} \sim\left|\chi_{c}\right| \vartheta_{2} \chi r^{3}=3 \frac{\left|\chi_{c}\right| P[\mathrm{~h}]}{R_{\odot}[\mathrm{AU}]^{2} r[\mathrm{~km}]^{2}} \mathrm{Myr}^{-1}$

is obtained with $P$ as the rotation period measured in hours, as indicated. One can define a YORP timescale $t_{Y}$ from this equation based on $|\dot{G} / G| t_{Y} \equiv 1$, which yields

$t_{Y}=0.3 \frac{R_{\odot}[\mathrm{AU}]^{2} r[\mathrm{~km}]^{2}}{\left|\chi_{c}\right| P[\mathrm{~h}]} \mathrm{Myr}$

where $\chi_{c}$ are radius normalized values like those of Eqs. (49), (54), and (55). The timescale is quite short, consistent with the studies of Komarov \& Sazonov (1994) and Sazonov (1994), and can be compared with the timescale due to the direct absorption of radiation, $t_{A}$ : From Mysen (2006), a small, uncertain value for $t_{A}$ is obtained if the 0.3 prefactor above is replaced by 10 and $\left|\chi_{c}\right|=1$, which is not that much longer than $t_{Y}$.

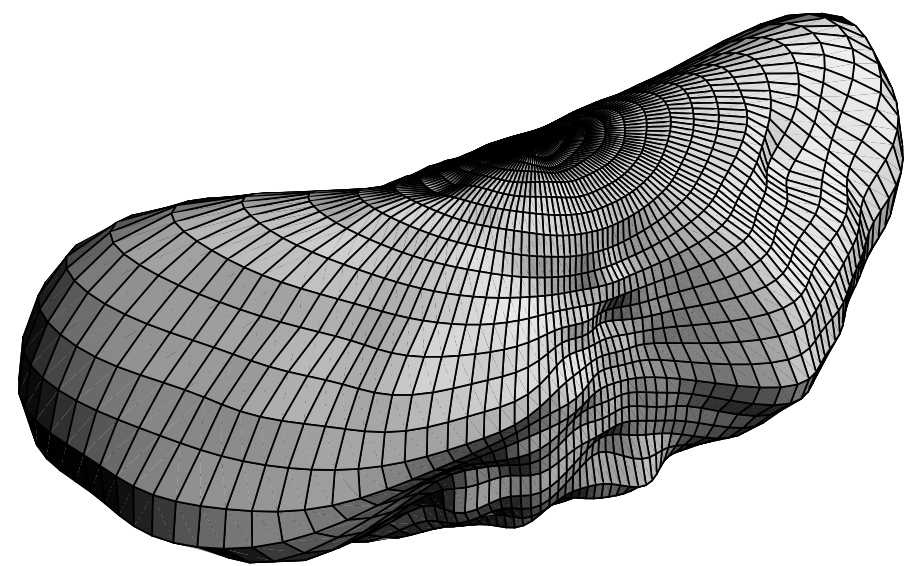

Fig. 7. The shape of the asteroid Eros from a spherical harmonics expansion up to and including order and degree 24.

\section{Comparison with previous work: Eros}

Before exploring the Yarkovsky effect in context of the presented formalism, so that we later can discuss the coupled rotational and translational dynamics of asteroids within a unified framework, we apply Eqs. (45) and (46) to the well-studied asteroid Eros. For this purpose, the spherical harmonics expansion of the asteroid's surface to degree and order 24 of Zuber et al. (2000), available at "http://www-geodyn.mit.edu/ near/nlr.30day.html", is used, plotted as Fig. 7.

Numerical integrations yield for the shape parameters of Eqs. (45) and (46)

$\chi_{s}=6.60 \times 10^{11} \mathrm{~m}^{3}, \quad \chi_{c}=5.75 \times 10^{10} \mathrm{~m}^{3}$

which, with a high $\bar{\lambda}_{t}=35$, shows that a delayed thermal response of this elongated and regolith-covered ellipsoidal surface causes the asteroid's spin axis to align itself with the orbit normal. From Miller et al. (2002), the spin or rotational angular momentum of Eros is

$G=C \omega=1.65 \times 10^{20} \mathrm{~kg} \mathrm{~m}^{2} \mathrm{~s}^{-1}$

where $C$ is the moment of inertia around the axis of rotation, and $\omega$ is the time derivative of the asteroid's rotational phase, as before. This yields for the prefactors of Eqs. (45) and (46) at heliocentric distance $R_{\odot}=2.5 \mathrm{AU}$ :

$\chi \vartheta_{2}=0.63 \times 10^{-27} \mathrm{~m}^{-3} \mathrm{~s}^{-1}$.

We include (Fig. 8) a plot of $\dot{\omega}=\omega \dot{G} / G$, according to Eqs. (45) and (58), where $\omega=1.19 \mathrm{radh}^{-1}$ for Eros. The units are chosen so that a comparison with the numerical studies of Capek \& Vokrouhlický (2004) can easily be made. The match is very good both qualitatively and quantitatively, considering the assumptions we make. There are three small differences. The two first are that $\dot{\omega}=0$ at $I \sim 55^{\circ}$ and $I \sim 125^{\circ}$, while in Čapek \& Vokrouhlický (2004) the change in rotation rate goes to zero when $I \sim 50^{\circ}$ and $I \sim 120^{\circ}$. Third, the curve in Fig. 8 lies in the range $\dot{\omega} \sim-1.9 \rightarrow 3.8$ in the given units, while in Čapek \& Vokrouhlický (2004) $\dot{\omega} \sim-1.7 \rightarrow 3.2$.

In Fig. 9, Eq. (46) is plotted for the shape parameters Eq. (58) and different $\bar{\lambda}_{1}$. This time the match with the numerical studies of Čapek \& Vokrouhlický (2004) seems to be even better than with the rotation rate comparison above. The transition from stable equilibria at $I \sim \pi / 2$ to stable equilibria at $I \sim 0$ and $I \sim \pi$ occurs at $\bar{\lambda}_{t}=35$, as previously stated. For a surface mass density of $\varrho=2 \mathrm{~g} \mathrm{~cm}^{-3}$ and heat capacity $C=680 \mathrm{~J} \mathrm{~kg}^{-1} \mathrm{~K}^{-1}$, which 


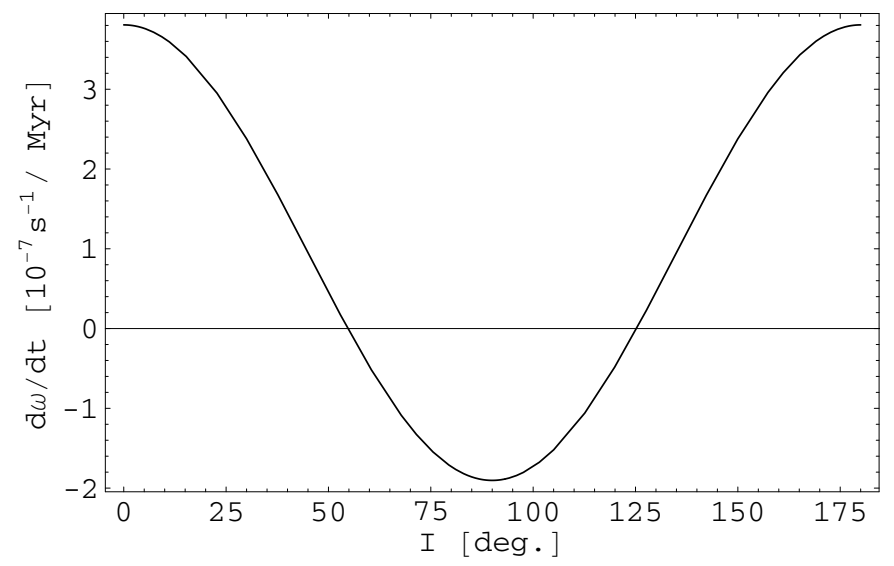

Fig. 8. The change in rotation rate as a function of obliquity according to the presented analytic theory is shown for Eros, Fig. 7.

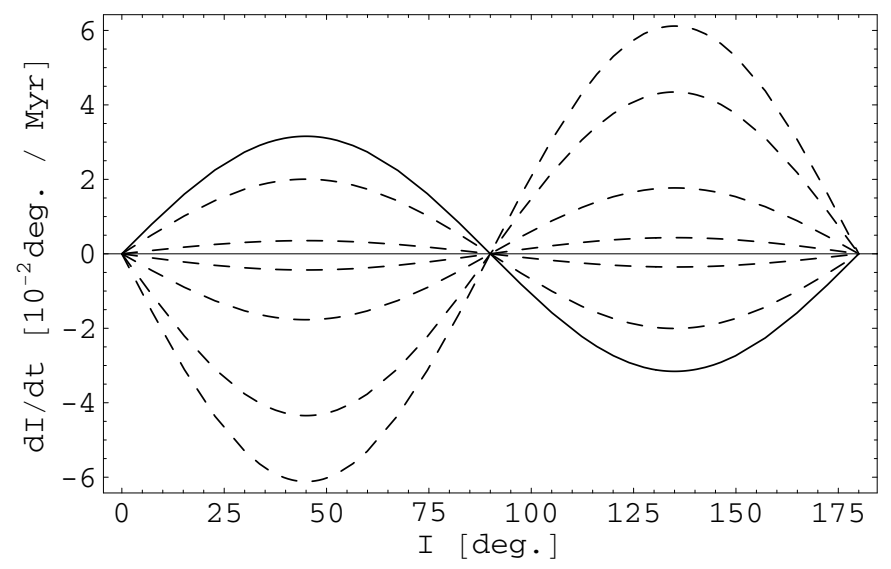

Fig. 9. The change in equatorial obliquity is plotted as a function of equatorial obliquity, according to the developed analytic theory. No heat conduction is represented by the solid line with $\bar{\lambda}_{1}=1000$. The dashed curves represent the cases $\bar{\lambda}_{1}=100,40$, a qualitative change occurs as predicted, then $\bar{\lambda}_{1}=30,20,10$, and at last $\bar{\lambda}_{1}=5$ for which the amplitude peaks.

are comparable to those used in the referenced studies (personal communication), this corresponds to a heat conductivity of

$K_{t}=5 \times 10^{-5} \mathrm{~W} \mathrm{~m}^{-1} \mathrm{~K}^{-1}$.

Although the value is only one step away, as measured in their resolution of heat conductivity, from the one given in Čapek \& Vokrouhlický (2004), namely $K_{t} \approx 5 \times 10^{-4} \mathrm{~W} \mathrm{~m}^{-1} \mathrm{~K}^{-1}$, the difference constitutes a discrepancy. However, it should be noted that Eq. (46) is sensitive to model simplifications when $K \sim K_{t}$, and the time derivative of the obliquity is close to zero. Accounting for these model simplifications could lead, therefore, to a significant correction of $\lambda_{t}$. According to Eq. (40), $K_{t} \sim \bar{\lambda}_{t}^{-2}$ where $\bar{\lambda}_{t}$ is a fairly sensitive function of $\lambda_{t}$, Eq. (43). That is, the value $K_{t}$ is sensitive to model errors. It is also possible that the simplification implied by the linearized approach Eq. (13) is of relevance here.

\section{Yarkovsky effect}

We have presented a formalism that consistently can describe all aspects related to the dynamical effects of thermal emission on asteroids. For such a complete description, the presented approach is here also applied to the characterization of the asteroid orbit evolution. In addition to providing a consistent framework for analysis of the coupled rotation and translation of asteroids, this study will also reveal whether there are translational effects connected to the irregularity of the asteroid's shape. Also, if the results presented below agree with previous work, we confirm the applied approach.

The time derivative of the asteroid orbit's semi-major axis is given by

$\dot{a}=\frac{2}{n}\left\langle\frac{C_{T}}{m_{a}}\right\rangle$

if the periodic terms are neglected, where the bracket is the same as the one of Eq. (24). Here $n$ is the rate of the asteroid's mean anomaly, $m_{a}$ the asteroid mass and $C_{T}$ is the force due to thermal emission along a unit vector $\boldsymbol{u}_{T}$ in the direction of the asteroid's motion. That is, if

$\boldsymbol{u}_{N}=\sum_{i} \gamma_{N, x_{i}} \boldsymbol{u}_{x_{i}}$

is a unit vector normal to the orbit plane with

$\gamma_{N, x_{i}}=\sum_{j} R_{j i} \gamma_{N, X_{i}}, \gamma_{N,(X, Y)}=0$, and $\gamma_{N, Z}=1$,

(see Fig. 1), the direction cosines of $\boldsymbol{u}_{T}$ along the principal axes of the asteroid are

$\gamma_{T, x_{i}}=\sum_{j, k} \varepsilon_{i j k} \gamma_{\odot, x_{j}} \gamma_{N, x_{k}}$

Now, we define the angles

$\theta_{2} \equiv \theta-u, \quad \theta_{3} \equiv \theta+u, \quad \theta_{4} \equiv u$,

which after some trigonometric manipulation yields

$\gamma_{T, x}=\sum_{i=2}^{3}\left(\Omega_{i}^{c} \cos \theta_{i}+\Omega_{i}^{s} \sin \theta_{i}\right)$

where the prefactors $\Omega_{i}^{c, s}$ are given in Appendix A. Likewise, it can be shown that

$\gamma_{T, y}=\gamma_{T, x}\left(\Omega_{i}^{c} \rightarrow \Omega_{i}^{s}, \Omega_{i}^{s} \rightarrow-\Omega_{i}^{c}\right)$

and

$\gamma_{T, z}=\Omega_{4}^{c} \cos \theta_{4}+\Omega_{4}^{s} \sin \theta_{4}$

These are needed for the evaluation of the contribution from a surface element to the secular drift in orbit size, see Eq. (20)

$$
\begin{aligned}
\mathrm{d}\left\langle C_{T}\right\rangle & =\sum_{i=1}^{3}\left\langle\gamma_{T, x_{i}} \mathrm{~d} F_{x_{i}}\right\rangle \\
& =-\frac{2}{3} \frac{\mathrm{d} S}{c} \Psi_{0}^{\prime} \sum_{i=1}^{3} \gamma_{x_{i}}\left\langle\gamma_{T, x_{i}} \Delta T\right\rangle
\end{aligned}
$$

In the same way as was done for the calculation of the obliquity alterations in Eq. (30), the thermally-delayed angles will be treated explicitly. That is

$w_{1} \rightarrow w_{i} \equiv \theta_{i}-\eta_{i}$ 
which yields

$$
\begin{array}{r}
\left\langle\Delta T\left(w_{i}\right) \gamma_{T, x}\right\rangle=S_{\odot} \sum_{i=2}^{3}\left[\frac{\cos \eta_{i}}{\sqrt{\beta_{i}^{2}+\Gamma_{i}^{2}}}\left(\Omega_{i}^{c} \epsilon_{i}^{c}+\Omega_{i}^{s} \epsilon_{i}^{s}\right)\right. \\
\left.-\frac{\sin \eta_{i}}{\sqrt{\beta_{i}^{2}+\Gamma_{i}^{2}}}\left(\Omega_{i}^{c} \epsilon_{i}^{s}-\Omega_{i}^{s} \epsilon_{i}^{c}\right)\right]
\end{array}
$$

from which $\left\langle\Delta T\left(w_{i}\right) \gamma_{T, y}\right\rangle$ can be calculated on basis of Eq. (68). The averages

$\epsilon_{i}^{c} \equiv\left\langle\epsilon \cos \theta_{i}\right\rangle, \quad \epsilon_{i}^{s} \equiv\left\langle\epsilon \sin \theta_{i}\right\rangle$

are included in Appendix B, with which one obtains

$$
\begin{aligned}
\sum_{i=1}^{2} \gamma_{x_{i}}\left\langle\Delta T \gamma_{T, x_{i}}\right\rangle & =-S_{\odot} \frac{\vartheta_{1}}{2}\left(\gamma_{x}^{2}+\gamma_{y}^{2}\right) \\
& \times\left[\frac{\sin \eta_{2}}{\sqrt{\beta_{2}^{2}+\Gamma_{2}^{2}}} \cos ^{4}(I / 2)-\frac{\sin \eta_{3}}{\sqrt{\beta_{3}^{2}+\Gamma_{3}^{2}}} \sin ^{4}(I / 2)\right]
\end{aligned}
$$

for the first terms of Eq. (70). If the rotation period of the asteroid is much smaller than its orbit period, one has

$\omega_{2} \approx \omega_{3} \approx \omega \equiv \dot{\theta} \Longrightarrow \Gamma_{2} \approx \Gamma_{3} \approx \Gamma_{1}$

and

$\sum_{i=1}^{2} \gamma_{x_{i}}\left\langle\Delta T \gamma_{T, x_{i}}\right\rangle=-S_{\odot} \frac{\vartheta_{1}}{2}\left(\gamma_{x}^{2}+\gamma_{y}^{2}\right) \frac{\Gamma_{1} \cos I}{\beta_{1}^{2}+\Gamma_{1}^{2}}$,

also known as the diurnal component. As for the last term of Eq. (70)

$\gamma_{z}\left\langle\Delta T \gamma_{T, z}\right\rangle=S_{\odot} \frac{\vartheta_{1}}{2} \gamma_{z}^{2} \frac{\Gamma_{4}}{\beta_{4}^{2}+\Gamma_{4}^{2}} \sin ^{2} I$,

which can be identified as the so-called seasonal Yarkovsky effect (Vokrouhlický 1999). It is then natural to introduce a thermal lag factor $\bar{\lambda}_{4}$, based on $\Gamma_{4}$, which contains the orbit frequency in the same way as $\bar{\lambda}_{1}$ is based on $\Gamma_{1}$, which contains the rotation frequency. At heliocentric distance $R_{\odot}=3 \mathrm{AU}$ we obtain for regolith-covered, basaltic, and metal-rich bodies, respectively

$\bar{\lambda}_{4}(R)=530, \quad \bar{\lambda}_{4}(B)=8.3, \quad$ and $\quad \bar{\lambda}_{4}(M)=1.4$,

(see Eqs. (51) and (52)). That is, both the basaltic and metal-rich body are in a regime where the seasonal component is effective, remembering that $\kappa_{1,4}^{s}$ has a maximum in the region $2 \lesssim \bar{\lambda}_{1,4} \lesssim$ 15. The skin depths of Eq. (19), which should be much smaller than the size of asteroid for the given approach to hold, are

$\zeta_{4}(R)=0.2 \mathrm{~m}, \quad \zeta_{4}(B)=6 \mathrm{~m}, \quad \zeta_{4}(M)=15 \mathrm{~m}$.

Finally,

$\left\langle C_{T}\right\rangle=\Xi \vartheta_{1}\left[\Xi_{1}(I) \cos I-\Xi_{4}(I) \sin ^{2} I\right]$,

where

$\Xi_{i}=\oint \mathrm{d} S\left(\gamma_{x}^{2}+\gamma_{y}^{2}\right) \kappa_{i}^{s}(I)$ with $\kappa_{i}^{s}$ corresponding to the function Eq. (42), but based on $\Gamma_{i}$ instead of $\Gamma_{1}$, and

$\Xi=\frac{1}{3} \frac{S_{\odot}}{c}$

Again, we use the approximation Eq. (43), and the structure, i.e., the dependency on the dynamical variables, of the Eq. (62) is clear:

$\dot{a}=\frac{2}{n} \frac{1}{m_{a}} \Xi \vartheta_{1} \Phi\left(\kappa_{1}^{s} \cos I-\kappa_{4}^{s} \sin ^{2} I\right)$

with

$\Phi=\oint \mathrm{d} S\left(\gamma_{x}^{2}+\gamma_{y}^{2}\right)$

However, this last approximation is not necessary if only the structure of the differential equation, rather than more precise representations, is of interest. That is, the integrand of Eq. (81) is always positive and a form of the differential equation similar to Eq. (83) follows.

According to the values Eqs. (50) and (78), regolith-covered bodies have their orbit sizes decreased for retrograde rotation, $\pi / 2<I<\pi$, and for prograde rotation, $0<I<\pi / 2$, the orbit semi-major axis increases. For metal-rich and basaltic asteroids the seasonal component is most effective and leads to a decrease in orbit size. As is demonstrated, there are no particular new dependencies for circular orbits related to an asteroid's shape irregularity.

The Gaussian shape of Fig. 4 yields

$\Phi=13.8$

while the ellipsoidal shape of Fig. 6 yields

$\Phi=6.86$.

Using a maximum $\kappa_{1,4}^{s} \sim 0.2$ and density $\varrho=2.5 \mathrm{~g} \mathrm{~cm}^{-3}$, we get the maximum

$\dot{a} \sim 0.03 \frac{\Phi}{R_{\odot}[\mathrm{AU}]^{1 / 2} r[\mathrm{~km}]} \mathrm{AU} \mathrm{Gyr}^{-1}$

with radius normalized values of $\Phi$ like those of Eq. (85) and (86).

The reader should take note of the fact that the exact form of Eq. (80) is valid independent of the included order $N$ of the insolation function's Fourier expansion

$\hat{\epsilon}=\sum_{i=0}^{N} \vartheta_{i} \cos ^{i} z$

which, regardless of the expanded order, contains only one component, $\vartheta_{i} \cos ^{i} z$, with $i$ odd, namely $i=1$. The reason for the completeness of Eq. (80) is, therefore, that $\gamma_{T, x_{i}} \sim \cos f$, as has been shown previously in this section. Now, since

$\Delta T \sim \vartheta_{i} \cos i f$

and the only contribution to the Yarkovsky effect is, after averaging Eq. (24), from the only odd term, $i=1$, of Eq. (88). 


\section{Discussion}

Asteroids absorb solar radiation, which they later emit at longer wavelengths, leading to a recoil force. We present an analytical theory that consistently models the effects of this thermal emission on an irregularly-shaped asteroid's orbit and rotation, here defined as the Yarkovsky and YORP effects, respectively. Furthermore, the important dynamical consequences of a delayed thermal response of the asteroid's surface to solar radiation are parameterized in terms of the fundamental properties of the asteroid's surface. The model is consistent with the overall results of previous numerical studies, and reproduces the obliquity equilibria and spin rates for the elongated and irregularly-shaped asteroid Eros.

To be more specific, the so-called diurnal Yarkovsky component is the most effective transport mechanism for regolithcovered bodies, and is maximized by a rotation axis normal to the orbit plane. The orbit sizes of metal-rich and basaltic asteroids are effectively decreased by the so-called seasonal Yarkovsky component which, on the other hand, vanishes for rotation axes aligned with the orbit normal.

It is previously known that a thermal lag of the asteroid surface can lead to an alignment of the rotation axis with the orbit normal. Here, it has been illustrated that the ellipsoidality of the asteroid shape is responsible for this effect. The delayed thermal response of a possibly irregular surface was connected directly to the physics of the problem, which enables a quantification of the transition from one type of obliquity equilibria to another in terms of the surface properties of the asteroid.

Since such a directly retrograde or prograde rotation optimizes the diurnal Yarkovsky effect, this represents a preference for the shapes of regolith-covered Earth orbit crossing asteroids. The mobility of ellipsoidal basaltic and metal-rich bodies in the main belt is minimized in the stable YORP equilibria of equatorial obliquity since the seasonal Yarkovsky effect vanishes. However, the timescale on which these YORP equilibria are reached is very long for metal-rich bodies, not only due to their larger densities. It is, therefore, questionable if the transport mechanisms of thermal emission lead to a preference for the shape of metal-rich Earth orbit crossing objects. This will, of course, be dependent on the location of such families with respect to the resonances of the main belt.

It should also be noted that according to lightcurves, asteroid rotation axes normal to the ecliptic seem to be favored (Pravec et al. 2002), although this may be due to a selection effect. The well-studied asteroid Eros, on the other hand, has a rotation axis that lies very close to its current orbit plane (Souchay et al. 2003). According to Vokrouhlický et al. (2005), this rotation state is more probable if it was the same as when the asteroid resided in the main belt, which is different from the thermal YORP obliquity equilibria.

At last, our model demonstrates clearly, with the differential equations' explicit dependencies on the dynamical variables, which effects are caused by corrections that are not included. That is, numerical studies of shapes with results that deviate from the simple structure presented are either caused by details of the interaction law, self-shadowing, or a thermal lag, which is not constant across the asteroid surface. It is also possible that the applied linearization of the problem's boundary condition can be the cause for some discrepancies in the presented dynamical tendencies. From the given approach and Mysen (2008), it should be possible to incorporate all of these corrections. It should be fairly straightforward to include the possibility of excited rotation and eccentric orbits. A pathological study of the important shape parameters in the presented theory would also be of interest.

Acknowledgements. This work was possible due to support from Kaare Aksnes and the Research Council of Norway, projects 170870 and 184686. The author thanks the anonymous referees for advice which improved the paper.

\section{Appendix A: Direction cosine components for calculation of Yarkovsky effect}

Some prefactors needed for the calculation of the secular drift of an asteroid's orbit size are listed below.

$$
\begin{aligned}
& \Omega_{2}^{c}=-\frac{1}{2}(1+\cos I) \sin h \\
& \Omega_{2}^{s}=-\frac{1}{2}(1+\cos I) \cos h \\
& \Omega_{3}^{c}=-\frac{1}{2}(1-\cos I) \sin h \\
& \Omega_{3}^{s}=\frac{1}{2}(1-\cos I) \cos h \\
& \Omega_{4}^{c}=\sin I \cos h \\
& \Omega_{4}^{s}=\sin I \sin h
\end{aligned}
$$

\section{Appendix B: Averages of the insolation function for the calculation of the Yarkovsky effect}

Below, averages needed for the calculation of the Yarkovsky effect are given.

$$
\begin{aligned}
& \epsilon_{2}^{c}=\frac{1}{4} \vartheta_{1}(1+\cos I)\left(\gamma_{x} \cos h-\gamma_{y} \sin h\right) \\
& \epsilon_{2}^{s}=-\frac{1}{4} \vartheta_{1}(1+\cos I)\left(\gamma_{y} \cos h+\gamma_{x} \sin h\right) \\
& \epsilon_{3}^{c}=\frac{1}{4} \vartheta_{1}(1-\cos I)\left(\gamma_{x} \cos h+\gamma_{y} \sin h\right) \\
& \epsilon_{3}^{s}=-\frac{1}{4} \vartheta_{1}(1-\cos I)\left(\gamma_{y} \cos h-\gamma_{x} \sin h\right) \\
& \epsilon_{4}^{c}=\frac{1}{2} \vartheta_{1} \gamma_{z} \sin I \sin h \\
& \epsilon_{4}^{s}=-\frac{1}{2} \vartheta_{1} \gamma_{z} \sin I \cos h
\end{aligned}
$$

\section{References}

Bottke, W. F., Vokrouhlický, D., \& Nesvorný, D. 2007, Nature, 449, 48 Breiter, S., Michalska, H., Vokrouhlický, D., \& Borczyk, W. 2007, A\&A, 471, 345

Burns, J., \& Safronov, V. 1973, MNRAS, 165, 403

Čapek, D., \& Vokrouhlický, D. 2004, Icarus, 172, 526

Efroimsky, M. 2001, Planet. Space Sci., 49, 937

Farinella, P., Vokrouhlický, D., \& Hartmann, W. K. 1998, Icarus, 132, 378

Fernandes, S. S., \& Sessin, W. 1989, Cel. Mech. Dyn. Astr., 46, 49

Goldstein, H. 1980, Classical Mechanics (Reading: Addison-Wesley)

Harris, A. W., \& Lagerros, J. S. V. 2002, in Asteroids III, ed. W. F. Bottke, A. Cellino, P. Paolicchi, \& R. P. Binzel (Tucson: The University of Arizona Press), 205

Komarov, M. M., \& Sazonov, V. V. 1994, Sol. Sys. Res., 28, 16

Miller, J. K., Konopliv, A. S., Antreasian, P. G., et al. 2002, Icarus, 155, 3 
Muinonen, K. 1998, A\&A, 332, 1087

Muinonen, K., \& Lagerros, J. S. V. 1998, A\&A, 333, 753

Mysen, E. 2006, MNRAS, 372, 1345

Mysen, E. 2007, MNRAS, 381, 301

Mysen, E. 2008, MNRAS, 383, L50

Neishtadt, A. I., Scheeres, D. J., Sidorenko, V. V., et al. 2002, Icarus, 157, 205

Neishtadt, A. I., Scheeres, D. J., Sidorenko, V. V., et al. 2003, Cel. Mech. Dyn. Astron., 86, 249

Nesvorný, D., \& Vokrouhlický, D. 2007, AJ, 134, 1750

Pravec, P., Harris, A. W., \& Michałowski, T. 2002, in Asteroids III, ed. W. F. Bottke, A. Cellino, P. Paolicchi, \& R. P. Binzel (Tucson: The University of Arizona Press), 113
Rubincam, D. P. 2000, Icarus, 148, 2

Sazonov, V. V. 1994, Sol. Sys. Res., 28, 152

Scheeres, D. J. 2007, Icarus, 188, 430

Skoglöv, E. 1996, Planet. Space Sci., 45, 439

Souchay, J., Kinoshita, H., Nakai, H., \& Roux, S. 2003, Icarus, 166, 285

Vokrouhlický, D. 1998, A\&A, 335, 1093

Vokrouhlický, D. 1999, A\&A, 344, 362

Vokrouhlický, D., \& Căpek, D. 2002, Icarus, 159, 449

Vokrouhlický, D., \& Nesvorný, D., \& Bottke, W. F. 2003, Nature, 425, 147

Vokrouhlický, D, Bottke, W. F., \& Nesvorný, D. 2005, Icarus, 175, 419

Zuber, M. T., Smith, D. E., Cheng, A. F., et al. 2000, Science, 289, 2097 